$$
\begin{aligned}
\mathbf{W}=\mathbf{r}_{1} A & \sin \frac{\theta}{\lambda v_{0}}\left(r \cos ^{\lambda} \frac{\theta}{\lambda v_{0}}\right)^{\left(v^{2} 0-1 / \lambda\right) /\left(1-v^{2} 0\right)} \\
& +\theta_{1} A v_{0} \cos \frac{\theta}{\lambda v_{0}}\left(r \cos ^{\lambda} \frac{\theta}{\lambda v_{0}}\right)^{\left(v^{2} 0-1 / \lambda\right) /\left(1-v^{2} 0\right)} \\
& +\mathbf{Z}_{1}\left[1-A^{2}\left(r \cos ^{\lambda} \frac{\theta}{\lambda v_{0}}\right)^{2\left(v^{2} 0-1 / \lambda\right) /\left(1-v^{2} 0\right)}\right]^{1 / 2},
\end{aligned}
$$

where $A$ is a constant such that

$$
0<A<\left(r \cos ^{\lambda} \frac{\theta}{\lambda v_{0}}\right)^{-\left(v^{2} 0-1 / \lambda\right) /\left(1-v^{2} 0\right)}
$$

throughout the flow region considered. The lines in the $r, \theta$ plane on which $r \cos ^{\lambda}\left(\theta / \lambda v_{0}\right)$ is equal to a constant (for fixed $v_{0}$ ) are the streamlines of the field (26). Therefore, for $v_{0}^{2}<1 / \lambda$ the innermost streamline in the region considered fixes the upper bound on $A$, and it is clear that the line $\theta=\lambda v_{0} \pi / 2$ must be excluded. For $v_{0}^{2}>1 / \lambda$ the point $r=\infty$ is the critical one in bounding $A$; only a finite part of the plane can be taken as the region considered.

\title{
BIBLIOGRAPHY
}

[1] H. Poritsky, Compressible flows obtainable from two-dimensional flows through addition of a constant normal velocity, A. S. M. E. Adv. Paper No. 45-A-26, 1945.

[2] M. Munk and R. Prim, On the multiplicity of steady gas flows having the same streamline pattern, Proc. Nat. Acad. Sci. 33, 137-141 (1947).

[3] R. Prim, A note on the substitution principle for steady gas flows, J. Appl. Phys. 20, 448-450 (1949).

[4] M. Munk and R. Prim, On the canonical form of the equations of steady motion of a perfect gas, J. Appl. Phys. 19, 957 (1948).

[5] P. Nemenyi and R. Prim, On the steady Beltrami flow of a perfect gas, Proc. 7th Internat. Cong. Appl. Mech., London, 1948.

[6] B. Hicks, P. Guenther, and R. Wasserman, New formulations of the equations for compressible flow, Q. Appl. Math. 5, 357-361 (1947).

[7] R. Prim, On a family of rotational gas flows, Q. Appl. Math. 6, 319-326 (1948).

\section{ON HEAT TRANSFER PROBLEMS IN VISCOUS FLOW*}

\author{
By G. F. CARRIER AND J. A. LEWIS (Brown University)
}

1. Summary. Many problems of physical interest which are associated with the flow of a viscous fluid through a narrow channel require the determination of the temperature distribution throughout the field of flow. In general, such problems may be separated into one of three classifications. The first of these is characterized by the existence of a thermal boundary layer, the second by a temperature distribution independent of the coordinate across the channel, and the third by an intermediate type

*Received March 7, 1949. 
of temperature distribution. In the present paper a simple method for the classification of such problems is given, and techniques for solving some of the problems which arise in practice are discussed.

2. The general equations. We shall consider the two-dimensional flow of an incompressible fluid with constant viscosity and heat conductivity. The equations governing this flow may be written

$$
\begin{gathered}
\rho\left(u u_{x}+v u_{y}\right)=-p_{x}+\mu \Delta u, \\
\rho\left(u v_{x}+v v_{y}\right)=-p_{y}+\mu \Delta v, \\
u_{x}+v_{y}=0,
\end{gathered}
$$

where $u, v$ are the velocity components in the $x, y$ directions, respectively, $p$ is the pressure, $\rho$ the density, $\mu$ the viscosity, and $\Delta$ the Laplacian operator.

To determine the temperature distribution, we also introduce the energy equation

$$
\rho c\left(u T_{x} \dot{+} v T_{y}\right)=k \Delta T+\mu\left[2 u_{x}^{2}+2 v_{y}^{2}+\left(u_{y}+v_{x}\right)^{2}\right],
$$

where $k$ is the heat conductivity coefficient, $c$ the heat capacity, and $T$ the absolute temperature.

3. Channel flow. We shall consider the flow of a viscous, heat-conducting fluid through a channel bounded by smooth walls of small curvature, which may be moving or stationary. Then Eqs. (1), (2), (3), and (4) may be replaced by a simplified set of equations, which are obtained here by making certain physically plausible assumptions concerning the nature of the flow. We assume that $u \gg v, p_{x} \gg p_{v}$, and that the Reynolds number of the flow is such that the inertia terms in the equations of motion are very small. Under these assumptions the general equations can be replaced by the approximate relations ${ }^{1}$

$$
\begin{gathered}
\mu u_{y y}=p_{x}=p^{\prime}(x), \\
\int_{-t_{2}}^{t_{2}} u(x, y) d y=\text { const., } \\
k T_{y y}-\rho c u T_{x}+\mu u_{y}^{2}=0,
\end{gathered}
$$

where $-t_{1}(x)$ and $t_{2}(x)$ are the coordinates of the upper and lower boundaries. Typical boundary conditions ${ }^{2}$ are

\footnotetext{
${ }^{1} \mathrm{~A}$ crude analysis will show that the term $\rho c v T_{y}$ is of the same order as $\rho c u T_{x}$. However, since the channel is assumed to be bounded by walls of small curvature, the streamlines of the flow will practically coincide with the lines $y=$ const. Therefore $v$ will be taken to be zero to the order of approximation considered here. An equation having the general form of Eq. (7) may be obtained also by a suitable change of variable. For example, in the doctoral thesis of M. Finston (Brown Univ.) the details of this variable change for a specific problem are given and it is shown that the approximation used here is valid.

${ }^{2}$ In general $T(0, y)$ is given only roughly for the following reason: the wall effects near the entrance imply a modification of the upstream temperature distribution via conduction and thus, at any given section, $T$ cannot be specified $a$ priori.
} 


$$
\begin{aligned}
& u\left(x,-t_{1}\right)=U_{1}, \\
& u\left(x, t_{2}\right)=U_{2}, \\
& p_{\text {inlet }}=\text { const., } \\
& T(0, y)=\text { const. }=T_{0}, \\
& T\left(x,-t_{1}\right)=T\left(x, t_{2}\right)=\text { const. }=T_{w} \text { or } \\
& \left(T_{y}\right)_{y=-t_{1}}=\left(T_{y}\right)_{y=t_{2}}=\text { const. }
\end{aligned}
$$

It is evident from the form of Eq. (5) that the velocity distribution at any section (i.e., at fixed $x$ ) is parabolic. That this is the velocity profile one obtains in such problems is well-known. Thus we need consider only Eq. (7) with its associated boundary conditions and regard the velocity $u$ as known.

It is convenient to make the definitions ${ }^{3}$

$$
\begin{aligned}
\xi & =x / L, \\
\eta & =\left[2 y+t_{1}(x)-t_{2}(x)\right] /\left[t_{2}(x)+t_{1}(x)\right], \\
\epsilon & =k L / \rho c U_{0} t_{0}^{2} \\
\theta & =\left(T-T_{0}\right) \rho c t_{0}^{2} / \mu U_{0} L,
\end{aligned}
$$

where $L$ is the length of the passage, $t_{0}$ the minimum half-width of the channel, and $U_{0}$ the maximum velocity at the section of minimum area. Equation (7) then becomes (to our order of approximation)

$$
\begin{gathered}
\epsilon \theta_{\eta \eta}-\left[\left(1-\eta^{2}\right) f(\xi)+U_{2}(1+\eta) / 2+U_{1}(1-\eta) / 2\right] \theta_{\xi} \\
+\left(U_{1}-U_{2}-4 \eta f\right)^{2} / 4=0
\end{gathered}
$$

where $f(\xi)$ is determined (in terms of $p^{\prime}(x), t_{2}(x)$, and $t_{1}(x)$ ) from Eqs. (5) and (6).

4. The characteristic parameter $\epsilon$. Let us now examine Eq. (8) with regard to the quantity $\epsilon$, which depends upon the material properties of the fluid, the velocity of flow, and the geometry of the channel.

If $\epsilon \gg 1$, Eq. (8) may be replaced by the approximate relation

$$
\theta_{\eta \eta}=0 .
$$

This implies that the temperature distribution across the channel, for $\epsilon \gg 1$, is independent of $\eta$. There will, of course, be a thin transition region near the inlet where this is not true, but to the order of our approximation we can consider the temperature distribution independent of the coordinate across the channel in this case.

When $\epsilon$ is of order one, it is more difficult to compute the temperature distribution, since now all the terms in the differential equation are of the same order. However, it is still required that the temperature distribution should change smoothly as the fluid progresses down the channel. Thus a numerical procedure can be employed with reasonable efficiency to obtain the temperature distribution for any particular problem. An example of this type of problem is the flow of lubricating oil through a bearing. "The

${ }^{3}$ The definition of $\eta$ is chosen to give $\eta= \pm 1$ at the passage walls.

${ }^{4}$ For a particular lubrication problem, it was found that $\epsilon \approx 4$. 
usual assumption of constant temperature across the oil film is not exactly satisfied; however, probably little error will result in any practical case where this assumption is made

When the heat conductivity of the fluid is small, the velocity of flow large, and the channel not too narrow, we will have $\epsilon \ll 1$. In this case we might expect that the first term of Eq. (8) could be neglected. However, if this is done, the solution found will never obey all the boundary conditions and in many cases will be singular at the walls. Hence, we must expect that the conductivity will play an important role near the walls. We shall anticipate that there will be a thermal boundary layer whose thickness is of order $t_{0} \epsilon^{m}$ in which the temperature will vary rapidly from its value at the wall to its value outside this layer. In the latter region the temperature is almost entirely governed by the convective effects. From a crude dimensional analysis we might conclude that $m \sim 1 / 2$. We shall see later, however, that the precise value of $m$ depends upon the boundary conditions imposed upon the velocity.

The solution of Eq. (8), with suitable boundary conditions, will be found as the sum of two solutions - a solution of the homogeneous equation which takes special account of the rapid variation of temperature near the walls and hence is a boundary layer solution, and a particular solution of the original inhomogeneous equation. Let us denote the boundary layer solution by $\theta_{1}$ and the particular solution by $\theta_{0}$. In general, the inhomogeneous solution will describe the convective contribution to the temperature field, whereas the homogeneous solution will describe the boundary layer effect. However, when one or both of the passage walls is stationary, even the inhomogeneous solution must be subdivided into convective and conductive parts, or else the solution will be found to be composed of such complicated functions that computation becomes impractical. This will be seen in the example at the end of the paper.

5. The boundary layer solution. To obtain the boundary layer solution, we consider the homogeneous equation

$$
\epsilon\left(\theta_{1}\right)_{\eta \eta}-F(\xi, \eta)\left(\theta_{1}\right)_{\xi}=0,
$$

where $F(\xi, \eta)$ is defined in Eq. (8). The flow near each wall is treated by itself, and the two boundary layer solutions thus found are combined to give a smooth fit at the middle of the channel. For example, suppose we consider the thermal boundary layer at the lower wall (i.e., at $\eta \approx-1$ ). We set

$$
\zeta=(\eta+1) / \epsilon^{m},
$$

and choose $m$ so that the convective and conductive terms arising from Eq. (9) under this substitution are of the same order in $\epsilon$. If only the terms of the lowest order are retained, $F$ may be written in the simplified form

$$
F=U_{1}+\epsilon^{m} \zeta\left[2 f(\xi)+\left(U_{2}-U_{1}\right) / 2\right] .
$$

If the wall is stationary, $U_{1}=0$. If it is moving, $U_{1}$ will be the dominant term near the wall and the term in $\epsilon^{m}$ can be neglected. In the first case we choose $m=1 / 3$ and in the second $m=1 / 2$. Then Eq. (9) has the form (with $p$ and $q$ known functions of $\xi$ )

if the wall is stationary, and

$$
\left(\theta_{1}\right)_{s \zeta}-\zeta p\left(\theta_{1}\right)_{\xi}=0,
$$

$$
\left(\theta_{1}\right)_{55}-q\left(\theta_{1}\right)_{\xi}=0,
$$


if the wall is moving. These equations may be reduced to a simpler form by the transformations

$$
\begin{aligned}
& \alpha=\int \frac{d \xi}{p(\xi)}, \\
& \alpha=\int \frac{d \xi}{q(\xi)},
\end{aligned}
$$

to the forms

$$
\begin{aligned}
& \left(\theta_{1}\right)_{55}-\zeta\left(\theta_{1}\right)_{\alpha}=0 \\
& \left(\theta_{1}\right)_{55}-\left(\theta_{1}\right)_{\alpha}=0 .
\end{aligned}
$$

The function $\theta_{1}$ must now satisfy boundary conditions of the following type:

$$
\begin{aligned}
& \theta_{1}=0, \text { for } \alpha=0 \text { (at the inlet); } \\
& \theta_{1} \rightarrow 0, \text { as } \zeta \rightarrow \infty \text { (at the middle of the channel); } \\
& \theta_{1}=G(\alpha), \text { for } \zeta=0,
\end{aligned}
$$

where $G$ is chosen so that $\theta_{0}+\theta_{1}$ satisfies the original boundary condition at the wall. The problem given at the end of the paper exemplifies the simple method of obtaining the boundary layer solutions by the application of the Laplace transform.

The determination of a particular solution depends so much upon the form of Eq. (8) in a particular problem that few statements can be made, in general, as to its form. In many cases it may be found by reducing the partial differential equation thus found to an ordinary differential equation by an appropriate change of variable. ${ }^{5}$

6. The flow between two parallel walls. To illustrate the method of solution when $\epsilon \ll 1$, we shall compute the temperature distribution in the flow of a viscous fluid between two parallel, stationary walls. The temperature of the fluid at the inlet will be taken as $T_{0}$ (although, as previously noted, this boundary condition will not be satisfied exactly), and the temperature of the wall will be taken as fixed at a value $T_{w}$. In this case, the function $F$ has the form

$$
F(\xi, \eta)=1-\eta^{2},
$$

and Eq. (8) becomes ${ }^{6}$ (rigorously in this example)

$$
\epsilon \theta_{\eta \eta}-\left(1-\eta^{2}\right) \theta_{\xi}+4 \eta^{2}=0 .
$$

By inspection, a particular solution of Eq. (12) is seen to be $-\eta^{4} / 3 \epsilon$. This solution, however, does not yield the proper behavior in the interior of the channel, where convection and dissipation are the dominant effects. The above solution takes into account conduction and dissipation, the effects of which are dominant only near the walls. We seek a solution which is of the order $\epsilon^{-1 / 3}$ at the walls and which tends to zero at the

${ }^{5}$ See, for example, M. Finston, Doctoral thesis, Brown Univ. (forthcoming).

${ }^{6}$ We note here that the exact solution of this problem could be expressed as an infinite series of certain integrals of Whittaker functions, but this fact is obviously of academic interest only. 
middle of the channel. To find such a solution, we split the particular solution $\theta_{0}$ into two parts, $\theta^{*}$ and $\theta^{* *}$, satisfying the equations

$$
\begin{aligned}
& \epsilon \theta_{\eta \eta}^{*}-\left(1-\eta^{2}\right) \theta_{\xi}^{*}=-4 \eta^{2}\left(1-\eta^{2 \nu-2}\right), \\
& \epsilon \theta_{\eta \eta}^{* *}-\left(1-\eta^{2}\right) \theta_{\xi}^{* *}=-4 \eta^{2 \nu},
\end{aligned}
$$

where $\nu$ is some large number. If now Eq. (13a) is replaced by the approximate relation

$$
\left(1-\eta^{2}\right) \theta_{\xi}^{*}=4 \eta^{2}\left(1-\eta^{2 v-2}\right),
$$

$\theta^{*}$ will be given by the expression

$$
\theta^{*}=4 \xi \eta^{2} \frac{1-\eta^{2 v-2}}{1-\eta^{2}}
$$

which vanishes for $\eta=0$ and approaches the value $4 \nu \xi$ for large $\nu$ as $\eta$ approaches \pm 1 . If we had merely neglected the first term in Eq. (12) and then computed the corresponding solution, we would have obtained a solution which becomes singular at $\eta= \pm 1$. This seemingly artificial device used above avoids this difficulty. To obtain a solution of Eq. (13b) we set

$$
\zeta= \begin{cases}\epsilon^{-1 / 3}(1+\eta), & \text { for }-1 \leqq \eta \leqq 0, \\ \epsilon^{-1 / 3}(1-\eta), & \text { for } 0 \leqq \eta \leqq 1 .\end{cases}
$$

If now we retain only the terms of lowest order in $\epsilon$, Eq. (13b) becomes

$$
\theta_{\zeta \zeta}^{* *}-2 \zeta \theta_{\xi}^{* *}=-4 \epsilon^{-1 / 3}\left(1-\epsilon^{-1 / 3} \zeta\right)^{2 \nu} .
$$

Since $\epsilon \ll 1$, a convenient choice for $\nu$ is $2 \nu=\epsilon^{-1 / 3}$. It is known that

$$
\lim _{\nu \rightarrow \infty}\left(1-\frac{\zeta}{2 \nu}\right)^{2 \nu}=e^{-\zeta},
$$

and thus, since we have assumed $\epsilon$ very small (i.e., $2 \nu$ very large), we may approximate Eq. (14) by

$$
\theta_{\zeta \zeta}^{* *}-2 \zeta \theta_{\xi}^{* *}=-4 \epsilon^{-1 / 3} e^{-\zeta} .
$$

An obvious solution to this equation is

$$
\theta^{* *}=-4 \epsilon^{-1 / 3} e^{-\zeta} .
$$

This solution tends to zero for large $\zeta$ (i.e., at the middle of the channel) and assumes the value $-4 \epsilon^{-1 / 3}$ at the wall. In terms of $\eta$ then the complete particular solution $\theta_{0}$ has the form

$$
\theta_{0}=4 \xi \eta^{2} \frac{\left(1-\eta^{2 \nu-2}\right)}{\left(1-\eta^{2}\right)}-8 \nu\left\{e^{-2 \nu(\eta+1)}+e^{-2 \nu(1-\eta)}\right\},
$$

where $2 \nu=\epsilon^{-1 / 3}$. This function takes on the approximate value $4 \nu(\xi-2)$ at the walls and exhibits the proper behavior at $\eta=0$. Furthermore, it practically vanishes all along the inlet section except near the walls.

To complete the determination of the temperature distribution, we must now obtain a boundary layer solution, $\theta_{1}$, which satisfies

$$
\left(\theta_{1}\right)_{\zeta 5}-2 \zeta\left(\theta_{1}\right)_{\xi}=0
$$


and the boundary conditions

$$
\begin{aligned}
& \theta_{1}(\xi, 0)=4 \nu(2-\xi)+\theta_{w} \\
& \theta_{1}(\xi, \infty)=0, \\
& \theta_{1}(0, \zeta) \simeq 0,
\end{aligned}
$$

where $\theta_{w}$ is the dimensionless wall temperature.

To obtain this solution, we set

$$
\theta_{1}(\xi, \zeta)=\int_{0}^{\infty} e^{-\xi t} g(t, \zeta) d t
$$

i.e., we assume that $\theta_{1}$ is the Laplace transform of some function $g$. Then $g$ must satisfy the differential equation

$$
g_{\zeta \zeta}(t, \zeta)+2 \zeta t g(t, \zeta)=0
$$

which has the solution

$$
g=A(t) \zeta\left(9 t / 2 \zeta^{3}\right)^{1 / 6} J_{1 / 3}\left(2\left(2 t \zeta^{3} / 9\right)^{1 / 2}\right)+B(t)\left(9 t / 2 \zeta^{3}\right)^{-1 / 6} J_{-1 / 3}\left(2\left(2 t \zeta^{3} / 9\right)^{1 / 2}\right) .
$$

If $A$ and $B$ are taken as constants, the function $\theta_{1}$ has the form, ${ }^{7}$

$$
\theta_{1}(\xi, \zeta)=A \zeta \xi^{-4 / 3} \exp \left(-2 \zeta^{3} / 9 \xi\right)+B \xi^{-2 / 3} \exp \left(-2 \zeta^{3} / 9 \xi\right) .
$$

The first term may be written as

$$
A \zeta \xi^{-4 / 3} \exp \left(-2 \zeta^{3} / 9 \xi\right)=3 A \frac{\partial}{\partial \xi} \int_{\zeta / \xi^{1 / 3}}^{\infty} \exp \left(-2 \alpha^{3} / 9\right) d \alpha .
$$

Equation (16) implies that if $\theta_{1}$ is a solution then $\left(\theta_{1}\right)_{\xi}$ is also a solution and vice versa. Hence $P(\xi, \zeta)$ is a solution, where

$$
P(\xi, \zeta)=\frac{(6)^{1 / 3}}{\Gamma(2 / 3)} \int_{\zeta / \xi^{1 / 3}}^{\infty} \exp \left(-2 \alpha^{3} / 9\right) d \alpha .
$$

The quantity $\Gamma(2 / 3)$ is the Gamma function with argument $2 / 3$. Note that $P(\xi, 0)=1$. It can easily be verified by direct differentiation that if $P$ is a solution of Eq. (16), a more general solution can be obtained by setting

$$
\theta_{1}(\xi, \zeta)=\int_{0}^{\xi} K(\xi-\tau) P(\tau, \zeta) d \tau
$$

where $K$ is an arbitrary function. In the example considered here we require that

$$
\theta_{1}(\xi, 0)=4 \nu(2-\xi)+\theta_{w} ;
$$

thus we set

$$
K(\xi-\tau)=-4 \nu
$$

a constant, and $\theta_{1}$ is given by

$$
\theta_{1}(\xi, \zeta)=\left(\theta_{w}+8 \nu\right) P(\xi, \zeta)-4 \nu \int_{0}^{\xi} P(\tau, \zeta) d \tau .
$$

${ }^{7}$ R. V. Churchill, Modern operational mathematics in engineering, McGraw-Hill Co., New York, 1944, p. 299. 
In terms of $\eta$ then the first approximation to the temperature distribution in this example has the form

$$
\begin{aligned}
\theta(\xi, \eta)=\left(\theta_{w}\right. & +8 \nu)\{P[\xi, 2 \nu(1+\eta)]+P[\xi, 2 \nu(1-\eta)]\} \\
& -4 \nu \int_{0}^{\xi}\{P[\tau, 2 \nu(1+\eta)]+P[\tau, 2 \nu(1-\eta)]\} d \tau \\
& -8 \nu\left[e^{-2 \nu(1+\eta)}+e^{-2 \nu(1-\eta)}\right]+4 \xi \eta^{2} \frac{1-\eta^{2 \nu-2}}{1-\eta^{2}}
\end{aligned}
$$

where $2 \nu=\epsilon^{-1 / 3}$.

\section{THE THICKNESS OF A SHOCK WAVE IN AIR*}

\section{By A. E. PUCKETT AND H. J. STEWART (California Institute of Technology)}

1. If the viscosity and heat conductivity of a gas are neglected, it is easy to show that a one-dimensional supersonic flow of this gas may be interrupted by a discontinuity, or shock wave, across which the velocity jumps to a subsonic value (see [1] and [2] $\dagger$ ). In a real gas, viscosity and heat conduction may be negligible through a large part of a supersonic flow field but clearly must become important in the neighborhood of the large velocity gradients associated with a shock wave. If these effects are considered in the equations of motion, it is seen that the deceleration corresponding to the velocity jump through a shock wave must actually occur in a finite, although short, distance.

The first theory of the thickness of a shock wave was given by Rayleigh [3] who assumed that the fluid was a thermally and calorically perfect gas, that the viscosity coefficient $\mu$ was constant and that the heat transfer coefficient $\lambda$ was zero. Taylor [1] considered the effect of a constant, non-zero value of $\lambda$ by assuming that the velocity jump across the shock wave was small compared with the local speed of sound. Becker [4] noticed that a solution could be obtained without linearization in the special case that the Prandtl number $\sigma=C_{P} \mu / \lambda=0.75$, where $C_{P}$ is the specific heat at constant pressure. In all of these investigations the physical constants, $C_{P}, \mu$ and $\lambda$, were considered to be constant, and the linear (Navier-Stokes) theories of the viscous stress tensor and the heat flux vector were used. All of these investigations showed the width of a shock wave in air at normal conditions to be extremely small; for strong shock waves the width was computed to be less than the molecular mean free path. In view of these results, several writers (see [5] and [6]) have discussed the influence of deviations from thermodynamic equilibrium and of the higher order, non-linear (Burnett) terms in the viscous stress tensor and heat flux vector.

In the present note the problem of the width of a shock wave in air is re-examined using a thermally perfect gas and the linear (Navier-Stokes) theories of the viscous stress tensor and the heat flux vector. It is found that the solution can be carried

*Received Dec. 13, 1948.

$\dagger$ Numbers in the brackets refer to the bibliography at the end of the paper. 